Dass bei den Direktverkäufen im Atelier Bedienstete eingesetzt worden sind, ergibt sich wiederum nicht nur aus den Zeugenaussagen vor dem Senat, sondern folgt ebenfalls aus dem Schweigen der Klägerin, bei der Kenntnisse auch hierzu vorauszusetzen sind. Auf einschlägige Aktivitäten gerade des Zeugen L. deutet schließlich der von der Klägerin nicht bestrittene Sachverhalt hin, dass der Künstler zahlreiche Bilder, die bei diesem Mitarbeiter im Zuge der Trennung von ihm zunächst beschlagnahmt worden waren, später für echt erklärt hat, ebenso wie er auf die weitere strafrechtliche Verfolgung verzichtet hat. Der bestrittene Vortrag der Klägerin, dem habe die Androhung von Prügel zugrunde gelegen, ändert nichts daran, dass es in der Verfügungsgewalt des Mitarbeiters L. entweder zahlreiche echte Bilder des Künstlers gegeben oder dass der Künstler dort zahlreiche unechte Gemälde belassen hat. Der Galerist des Künstlers hat vor dem Landgericht in Bezug auf etwaige
Fälschungen auf eine gewisse „Unruhe“ im Atelier in den neunziger Jahren verwiesen. Im Übrigen hat der Zeuge von einer „'riesigen Woge' von dubiosen Bildern" gesprochen.

Dass der Bruder des Beklagten beim Kauf des Gemäldes ein allfälliges Fehlen der Befugnis des Mitarbeiters L. gekannt hätte, was die Anwendung bürgerlich-rechtlicher Rechtsscheinvorschriften des Vertretungsrechts sowie der allgemeinen Grundsätze des Schutzes von Treu und Glauben ausschließen würde, behauptet die Klägerin nicht einmal. Die Beweislast hierfür läge bei ihr (vgl. Palandt-Ellenberger, BGB, 73. Aufl., § 173 Rn. 2).

Die Revision ist nicht zuzulassen, weil die Entscheidung auf der Würdigung der Umstände des Einzelfalls beruht. (Entscheidung von der Redaktion bearbeitet.)

\title{
Umsatzsteuerrechtliche Erfüllung der gleichen kulturellen Aufgaben wie ein Museum in öffentlich-rechtlicher Trägerschaft
}

Bundesverwaltungsgericht, Beschluss vom 9. Juli 2014 - BVerwG 9 B 63.13

(Vorinstanzen: Verwaltungsgericht Köln, Urteil vom 30. Januar 2013 - VG 24 K 4102/09; Oberverwaltungsgericht

Nordrhein-Westfalen, Urteil vom 31. Juli 2013 - OVG 14 A 457/13*)

Die zuständige Landesbehörde kann im Bescheinigungsverfahren nach $\$ 4$ Nr. 20 Buchst. a S. 2 UStG ihre Prüfung, ob eine Einrichtung die gleichen kulturellen Aufgaben wie ein Museum in öffentlich-rechtlicher Trägerschaft erfüllt, nicht losgelöst vom Museumsbegriff in $\S 4$ Nr. 20 Buchst. a S. 5 UStG vornehmen, d.h. sie muss beachten, dass Museen im Sinne dieser Vorschrift „wissenschaftliche Sammlungen und Kunstsammlungen“sind. Die abschließende Entscheidung, ob eine Kunst- und Ausstellungshalle, die unstreitig nicht über eine eigene Sammlung verfügt, aber u.a. fremde Sammlungen als „Kunstausstellung“ präsentiert, eine „vergleichbare Einrichtung“ eines „anderen Unternehmers“ iSd § 4 Nr. 20 Buchst. a S. 2 USt ist, bleibt der nachfolgenden eigenständigen Prüfung durch die Finanzverwaltung vorbehalten. (Amtlicher Leitsatz)

\section{Gründe}

- Die auf die Zulassungsgründe des $\S 132$ Abs. 2 Nr. 1 und 3 VwGO gestützte Beschwerde bleibt ohne Erfolg.

1. Die Verfahrensrüge ( $\$ 132$ Abs. 2 Nr. 3 VwGO) greift nicht durch.

Der von der Klägerin als unzureichend beanstandete Hinweis im Berufungsurteil, die Voraussetzungen des § 132 Abs. 2 VwGO für eine Zulassung der Revision seien nicht erfüllt, genügt den Anforderungen an eine Begründung der Entscheidung über die Nichtzulassung der Revision (stRspr; vgl. nur Beschluss v. 30. Juli 1990 - BVerwG 7 B $104.90=$ Buchholz 310 § 132 VwGO Nr. 289 unter Hinweis auf Beschluss v. 13. Oktober 1967 - BVerwG 4 C 87.66). Im Übrigen wird hiermit ein Verfahrensmangel iSv § 132 Abs. 2 Nr. 3 VwGO schon deshalb nicht dargetan, weil nach dieser

Veröffentlicht in KUR 2013, 192.
Vorschrift nur solche Verfahrensmängel gerügt werden können, die der Entscheidung des Berufungsgerichts zur Sache anhaften (Beschluss v. 30. Juli 1990 aaO unter Hinweis auf Beschluss v. 12. Juni 1989 - BVerwG 7 B 123.88 = Buchholz 415.1 AllgKommR Nr. 88 ; Beschluss v. 15. Mai 2014 - BVerwG 9 B 57.13 [...] Rn. 22).

2. Die Rechtssache hat auch nicht die grundsätzliche Bedeutung (§ 132 Abs. 2 Nr. 1 VwGO), die ihr die Beschwerde beimisst.

Grundsätzlich bedeutsam iSv § 132 Abs. 2 Nr. 1 VwGO ist eine Rechtssache nur dann, wenn in dem angestrebten Revisionsverfahren die Klärung einer bisher höchstrichterlich ungeklärten, in ihrer Bedeutung über den der Beschwerde zugrunde liegenden Einzelfall hinausgehenden, klärungsbedürftigen und entscheidungserheblichen Rechtsfrage des revisiblen Rechts ( $\$ 137$ Abs. 1 VwGO) zu erwarten ist. Aus den Darlegungen der Beschwerde ergibt sich nicht, dass diese Voraussetzungen hier erfüllt sind. 
a) Der Frage, ob die Kultusbehörde im Rahmen ihrer Prüfung nach § 4 Nr. 20 Buchst. a S. 2 UStG auch prüfen muss, ob eine in der Rechtsform einer GmbH geführte Einrichtung, deren alleiniger Gesellschafter der Bund und die 16 Bundesländer sind, als "anderer Unternehmer" anzusehen ist und folglich keine Einrichtung einer öffentlichen Körperschaft nach § 4 Nr. 20 Buchst. a S. 1 UStG sein kann, kommt mangels Klärungsbedürftigkeit eine grundsätzliche Bedeutung nicht zu, denn sie ist in der obergerichtlichen Rechtsprechung bereits geklärt. Danach obliegt die abschließende Beurteilung, ob der Unternehmer eine Einrichtung betreibt, die einer Einrichtung iSd § 4 Nr. 20 Buchst. a S. 1 UStG gleichartig ist, nicht den für die Erteilung der Bescheinigung zuständigen Landesbehörden, sondern den Finanzbehörden bzw. im Streitfall den Finanzgerichten. Demgegenüber stellen die zuständigen Landesbehörden im Rahmen ihrer Prüfung nach § 4 Nr. 20 Buchst. a S. 2 UStG (lediglich) fest, ob eine Einrichtung die gleichen kulturellen Aufgaben wie die in $\S 4$ Nr. 20 Buchst. a S. 1 UStG bezeichneten Einrichtungen erfüllt. Hierdurch macht sich die Finanzbehörde das Fachwissen der zuständigen Kultusbehörde nutzbar (stRspr; vgl. nur BFH, Urteile v. 20. April 1988 - X R 20/82; BFHE 153, 454 (457 f.), v. 4. Mai 2011 - XI R 44/08; BFHE 233, 367 Rn. 15 und v. 19. Oktober 2011 - XI R 40/09 [...] Rn. 25; BVerwG, Urteile v. 4. Mai 2006 - BVerwG 10 C 10.05 = Buchholz 401.2 § 4 UStG Nr. 2 Rn. 21, v. 11. Oktober 2006 - BVerwG 10 C $7.05=$ Buchholz 401.2 $\$ 4$ UStG Nr. 3 Rn. 19 ff., v. 11. Oktober 2006 - BVerwG 10 C 4.06 = Buchholz 401.2 § 4 UStG Nr. 4 Rn. 13 ff. sowie Beschluss v. 31. Juli 2008 - BVerwG 9 B 80.07 = Buchholz 401.2 § 4 UStG Nr. 5 Rn. 4). Die Bescheinigung ist eine materiell-rechtliche Voraussetzung für die Steuerbefreiung durch die Finanzbehörden (BFH, Beschluss v. 16. August 1993 - V B 47/93 [...] Rn. 7).

Die Klägerin zeigt keine neuen, bislang nicht berücksichtigten rechtlichen Gesichtspunkte auf, die Anlass zu einem Überdenken dieser Rechtsprechung geben könnten.

Durch den Hinweis auf das Urteil des Verwaltungsgerichts Frankfurt v. 27. Juni 2012 - 6 K 2133/11.F (NJW 2013, 807) wird die gefestigte obergerichtliche Rechtsprechung, die entgegen der Darstellung in der Beschwerde auch in der rechtswissenschaftlichen Literatur nicht geteilt wird (vgl. nur Offerhaus/ Söhn/Lange-Kossack, UStG, Stand April 2014, § 4 Nr. 20 Rn. 9 ff.; Hartmann/Metzenmacher-Verweyen, UStG, 7. Aufl., 2011, E § 4 Nr. 20 Rn. 67 ff. und Stadie, UStG, 2. Aufl., 2012, § 4 Nr. 20 Rn. 10), nicht in Zweifel gezogen. Zwar hat das Verwaltungsgericht in der zitierten Entscheidung bei der Prüfung der Umsatzsteuerbefreiung für einen Kunstverein, der in seinen Räumen u.a. Kunstausstellungen durchführt, entgegen der oben beschriebenen Zuständigkeitsverteilung nicht nur die Wahrnehmung gleicher kultureller Aufgaben, sondern auch das Vorliegen einer "gleichartigen Einrichtung" geprüft; hierbei hat es Fragen der Zuständigkeit aber nicht einmal angesprochen. Insofern kann die Entscheidung nicht als Beleg dafür dienen, dass die aufgeworfene Frage „in der bisherigen verwaltungsgerichtlichen Rechtsprechung umstritten" ist, wie die Beschwerde vorträgt.
Auch der weitere in der Beschwerde enthaltene Hinweis auf die unterschiedlichen Rechtsauffassungen zu der Frage, ob nur Einrichtungen in der Rechtsform einer Körperschaft des öffentlichen Rechts unter $\S 4$ Nr. 20 Buchst. a S. 1 UStG fallen, oder ob diese Einrichtungen auch dann Steuerfreiheit genießen, wenn Bund, Länder oder Gemeinden sich der Rechtsform privatrechtlicher Gesellschaften bedienen, zeigt in Bezug auf die oben näher beschriebene Rechtsprechung zur Prüfung der tatbestandlichen Voraussetzungen der Steuerbefreiung keinen Klärungsbedarf auf. Bei dem Meinungsstreit, den das Oberverwaltungsgericht in seinem Urteil näher dargestellt hat, geht es allein darum, ob sich die Steuerfreiheit für privatrechtliche Gesellschaften in öffentlich-rechtlicher Trägerschaft aus $\S 4 \mathrm{Nr}$. 20 Buchst. a S. 1 oder aus $\S 4 \mathrm{Nr} .20$ Buchst. a S. 2 UStG ergibt. Hierdurch wird aber nicht in Frage gestellt, dass die Prüfung sämtlicher Tatbestandsmerkmale des $\S 4$ Nr. 20 Buchst. a S. 2 UStG ("gleichartige Einrichtungen anderer Unternehmer") mit Ausnahme des Merkmals der "gleichen kulturellen Aufgabenerfüllung" der beschriebenen Aufteilung zufolge allein in die Zuständigkeit der Finanzbehörden bzw. der Finanzgerichte fällt.

Die weiteren Argumente der Beschwerde, insbesondere die sich teilweise überschneidenden Gleichartigkeitsprüfungen und der Gesichtspunkt der Verwaltungspraktikabilität, geben ebenfalls keinen Anlass, die aufgezeigte Rechtsprechung zu überdenken; sie enthalten keine neuen Erwägungen. Dass im Einzelfall „praktische Schwierigkeiten“ auftreten können, ist zwar nicht zu leugnen; diese Schwierigkeiten sind aber überwindbar, ohne dass die Kultusverwaltung mit einer abschließenden Beurteilung auch sämtlicher Fragen belastet werden muss, die sich im Rahmen der den Finanzbehörden zugewiesenen "Gleichartigkeitsprüfung" zusätzlich ergeben können (vgl. hierzu bereits Urteil v. 11. Oktober 2006 - BVerwG 10 C 4.06 = Buchholz 401.2 $\S 4$ UStG Nr. 4 Rn. 16). Ebenso wenig ist es geboten, die der Finanzverwaltung obliegende "Gleichartigkeitsprüfung" als für das Bescheinigungsverfahren - wie die Klägerin offenbar meint - denkgesetzlich vorrangig oder sonst "vorgreiflich" einzustufen (so schon Urteil v. 11. Oktober 2006 - BVerwG 10 C $7.05=$ Buchholz 401.2 § 4 UStG Nr. 3 Rn. 22). Entscheidend ist, dass die Zuständigkeitsabgrenzung so klar ist, dass eindeutig feststeht, welche Behörde abschließend über welche Tatbestandsmerkmale zu entscheiden hat. Das ist der Fall.

b) Hiervon ausgehend ist die Revision auch nicht zur Klärung der Frage, in welchem Umfang die zuständige Landesbehörde bei der Bescheinigung der gleichen kulturellen Aufgabenerfüllung nach $\S 4$ Nr. 20 Buchst. a S. 2 UStG auf die Museumsdefinition des Satzes 5 Bezug nehmen muss, zuzulassen. Diese Frage ist, soweit sie einer abstrakten Klärung zugänglich ist, in der Rechtsprechung des Bundesverwaltungsgerichts bereits beantwortet. So wurde bereits entschieden, dass die Kultusbehörde nicht mit Bindungswirkung für die Finanzverwaltung darüber zu befinden hat, ob es sich bei der Einrichtung um ein Museum oder um eine sonstige in $\S 4 \mathrm{Nr}$. 20 Buchst. a S. 1 UStG genannte Einrichtung handelt bzw. ob das Unternehmen eine Einrichtung 
betreibt, die einer der dort genannten Einrichtungen, wie Museen in öffentlicher Trägerschaft, gleichartig ist. Der Umstand, dass sich die beiden - einerseits der Kultusbehörde, andererseits der Finanzverwaltung obliegenden - „Gleichartigkeitsprüfungen" inhaltlich teilweise überschneiden, führt allerdings dazu, dass die Entscheidung der Kultusbehörde darüber, ob die betreffende Einrichtung die gleichen kulturellen Aufgaben wie ein Museum in öffentlich-rechtlicher Trägerschaft erfüllt, nicht losgelöst vom Museumsbegriff in $\S 4$ Nr. 20 Buchst. a S. 5 UStG getroffen werden kann. Die Kultusbehörde hat also zu beachten, dass Museen danach „wissenschaftliche Sammlungen und Kunstsammlungen" sind, und sie muss prüfen, ob die in Rede stehende Einrichtung alle Voraussetzungen erfüllt, die nach dieser Legaldefinition für Museen unter dem Blickwinkel ihrer kulturellen Aufgabenerfüllung kennzeichnend sind (vgl. Urteil v. 11. Oktober 2006 - BVerwG 10 C 7.05, aaO Rn. 20 ff.) Die abschließende Entscheidung, ob die betreffende Einrichtung - hier eine Kunst- und Ausstellungshalle, die nicht über eine eigene Sammlung verfügt, aber u.a. fremde Sammlungen als „Kunstausstellung" präsentiert (vgl. Verweyen, aaO E § 4 Nr. 20 Rn. 42 und Kossack, aaO $\$ 4$ Nr. 20 Rn. 18) - eine "vergleichbare Einrichtung" eines „anderen Unternehmers“ iSd § 4 Nr. 20 Buchst. a S. 2 UStG ist, bleibt der nachfolgenden eigenständigen Prüfung durch die Finanzverwaltung vorbehalten. Von diesen Grundsätzen hat sich auch das Oberverwaltungsgericht leiten lassen. Einen darüber hinausgehenden fallübergreifenden Klärungsbedarf zeigt die Beschwerde nicht auf.

c) Schließlich rechtfertigt auch die Frage, ob die Ablehnung der verwaltungsgerichtlichen Überprüfung des Vorliegens der Tatbestandsvoraussetzungen in $\S 4$ Nr. 20 Buchst. a S. 2 UStG („gleichartige Einrichtung“ eines „anderen Unternehmers“) bei gleichzeitiger Verweisung der Klägerin auf die Möglichkeit ihrer finanzgerichtlichen Überprüfung eine Rechtsschutzlücke entstehen lässt, soweit sie einer abstrakten Beantwortung zugänglich ist, nicht die Zulassung der Revision.

Die Beschwerde steht auf dem Standpunkt, das Oberverwaltungsgericht hätte als Gericht des zulässigen Rechtsweges nach § 17 Abs. 2 S. 1 GVG über die beiden in der aufgeworfenen Frage genannten Tatbestandsmerkmale mitentscheiden müssen; stattdessen sei die Klägerin "rechtsverweigernd“ darauf verwiesen worden, in derselben Angelegenheit einen neuen Rechtsstreit vor den Finanzgerichten zu beginnen. Damit verkennt sie die Bedeutung des $§ 17$ Abs. 2 S. 1 GVG. Nach dieser Vorschrift entscheidet das Gericht des zulässigen Rechtsweges "den Rechtsstreit" unter allen in Betracht kommenden rechtlichen Gesichtspunkten. Dies setzt voraus, dass Gegenstand des Verfahrens ein einheitlicher Streitgegenstand im Sinne eines einheitlichen prozessualen Anspruchs ist. Liegt hingegen eine Mehrheit prozessualer Ansprüche vor, ist für jeden dieser Ansprüche die Rechtswegzuständigkeit gesondert zu prüfen (einhellige Auffassung, vgl. nur $B G H$, Beschluss v. 27. November 2013 - III ZB 59/13, BGHZ 199, 159 Rn. 14; Schoch/Schneider/Bier-Ehlers, VwGO, Stand April 2013, § 17 GVG § 41 Rn. 24; Eyermann-Rennert, VwGO, 13. Aufl., 2010, § 41/§§ 17-17b GVG
Rn. 18 f. mwN). Nach dem sogenannten zweigliedrigen Streitgegenstandsbegriff wird der Streitgegenstand im Allgemeinen als der prozessuale Anspruch durch die erstrebte, im Klageantrag umschriebene Rechtsfolge und den Klagegrund, d.h. den Sachverhalt, aus dem sich die Rechtsfolge ergeben soll, gekennzeichnet (stRspr; siehe etwa Urteile v. 31. August 2011 - BVerwG 8 C 15.10, BVerwGE 140, 290 Rn. 20 = Buchholz 428 § 6 VermG Nr. 75 und v. 10. Mai 1994 - BVerwG 9 C 501.93, BVerwGE 96, 24 (25) = Buchholz 310 § 121 VwGO Nr. 68 S. 2; Beschluss v. 20. September 2012 - BVerwG 7 B 5.12 [...]). Hiervon ausgehend handelt es sich bei der im vorliegenden Fall umstrittenen Aufhebung der von der zuständigen Landesbehörde ausgestellten Bescheinigung nach $\S 4$ Nr. 20 Buchst. a S. 2 UStG einerseits und dem Streit um die Entscheidung der Finanzbehörde über die Steuerfreiheit nach § 4 Nr. 20 Buchst. a S. 1 und 2 UStG andererseits um zwei unterschiedliche Streitgegenstände, sodass § 17 Abs. 2 S. 1 GVG keine Anwendung findet.

Auch die von der Klägerin behauptete Rechtsschutzlücke ist nicht erkennbar. Der zuständigen Landesbehörde kommt im Rahmen der Prüfung nach § 4 Nr. 20 Buchst. a S. 2 UStG - wie oben näher ausgeführt - lediglich die Aufgabe zu, die Gleichartigkeit der kulturellen Aufgabenwahrnehmung zu bescheinigen, wobei im Falle eines Museums die Legaldefinition des § 4 Nr. 20 Buchst. a S. 5 UStG zu beachten ist. Dementsprechend kann der Steuerpflichtige mit dem Einwand, er betreibe kein Unternehmen, das einer der in $\S 4 \mathrm{Nr}$. 20 Buchst. a S. 1 UStG genannten Einrichtungen gleichartig sei, nicht eine Entscheidung dieser Frage im Bescheinigungsverfahren oder im nachfolgenden Verwaltungsprozess erzwingen; denn diese Entscheidung obliegt ausschließlich dem Finanzamt und im Streitfall dem Finanzgericht (Urteil v. 11. Oktober 2006 - BVerwG 10 C 4.06, aaO Rn. 14 mwN aus der Rechtsprechung des BFH). Daher bleibt es einer Einrichtung unbenommen, im Rahmen des Steuerverfahrens gegenüber der Finanzbehörde geltend zu machen, dass sie aufgrund ihrer besonderen Organisationsstruktur oder wegen sonstiger Besonderheiten weder eine Einrichtung in öffentlicher Trägerschaft iSd § 4 Nr. 20 Buchst. a S. 1 UStG noch eine gleichartige Einrichtung nach § 4 Nr. 20 Buchst. a S. 2 UStG darstellt, also nicht umsatzsteuerbefreit ist. Darüber hinaus kann sie vorbringen, dass bestimmte Umsätze, die nicht eng mit dem „Kulturbetrieb" der betreffenden Einrichtung zusammenhängen, nicht steuerbefreit sind (siehe auch Kossack, aaO, § 4 Nr. 20 Rn. 21 und 35, wonach bei einem Museum zu den begünstigten Umsätzen etwa Ausstellungen, Führungen und Vorträge sowie der Verkauf von Katalogen und Museumsführern gehören, nicht aber Vermietungsumsätze oder die Veranstaltung von Kunstausstellungen für Dritte; vgl. zur Abgrenzung auch die Verwaltungsregelung zur Anwendung des Umsatzsteuergesetzes - UStAE 4.20.3. Abs. 3). (Entscheidung von der Redaktion bearbeitet.) 